\title{
Gluon versus sea quark shadowing
}

\author{
N. Hammon, H. Stöcker, W. Greiner ${ }^{1}$ \\ Institut für Theoretische Physik, Robert-Mayer Str. 10, Johann Wolfgang Goethe-Universität, 60054 Frankfurt am Main, Germany
}

Received 7 October 1998; revised 12 January 1999

Editor: P.V. Landshoff

\begin{abstract}
We calculate the shadowing of sea quarks and gluons and show that the shadowing of gluons is not simply given by the sea quark shadowing, especially at small $x$. The calculations are done in the lab frame approach by using the generalized vector meson dominance model. Here the virtual photon turns into a hadronic fluctuation long before the nucleus. The subsequent coherent interaction with more than one nucleon in the nucleus leads to the depletion $\sigma\left(\gamma^{*} A\right)<A \sigma\left(\gamma^{*} N\right)$ known as shadowing. A comparison of the shadowing of quarks to E665 data for ${ }^{40} \mathrm{Ca}$ and ${ }^{207} \mathrm{~Pb}$ shows good agreement. (C) 1999 Published by Elsevier Science B.V. All rights reserved.
\end{abstract}

\section{Introduction}

When calculating perturbative QCD cross sections in nucleus nucleus collisions one has to take care of an additional effect not appearing on the pure nucleon nucleon level: nuclear shadowing [1-3]. As a result of this depletion of the nuclear parton densities at small $x$ one finds a strong suppression of e.g. charmonium states and minijets [4,5]. Also for prompt photons smaller multiplicities result [6] due to the smaller number density of partons in the relevant region of the momentum fraction variable.

In either model (lab frame vector meson dominance type models [7] or infinite momentum frame parton fusion [8] models) the shadowing of gluons is expected to be much stronger than the shadowing of sea quarks, even at small $x$ [9-11]. This seems to

\footnotetext{
${ }^{1}$ Work supported by BMBF, DFG, GSI.
}

contradict the first naive expectation in terms of the QCD improved parton model that explains the scaling violation of the structure functions via the DGLAP splitting functions [12] which treat sea quarks similar to gluons (in the sense that the sea quarks are produced by the gluons when $Q^{2}$ increases) in a region where essentially no momentum is carried by valence quarks, i.e. in the small $x$ region.

In the following we will focus on the lab frame interpretation which explains the shadowing phenomenon by use of the generalized vector meson dominance model (GVMD).

\section{Lab frame description of shadowing}

In the lab frame description one essentially makes use of the hadronic structure of the virtual photon, manifesting itself in a field theoretic approach in two 
different time orderings assuring gauge- and Lorentz invariance to the amplitudes [13]. At small enough $x(x \ll 0.1)$ the hand-bag graph contribution gets small. The interaction then proceeds via the VMD graph where the virtual photon fluctuates into a $q \bar{q}$ pair within the "coherence time" $\lambda \approx 2 \nu /\left(\mu^{2}+\right.$ $\left.Q^{2}\right) \approx 1 /(2 m x)$, where $m$ is the nucleon mass, $\mu$ the mass of the pair and $\nu$ the lepton energy loss. At small $Q^{2}$ the interaction with a free nucleon then proceeds via the $\rho, \omega$, and $\phi$ mesons. At larger $Q^{2}$ the $q \bar{q}$ continuum has to be taken into account to describe the $F_{2}^{N}\left(x, Q^{2}\right)$ data. Including sea quarks $x f_{N}\left(x, Q^{2}\right)$ only, the nucleon structure function can (for small $x$ ) be written as [14]

$F_{2}^{N}\left(x, Q^{2}\right)=\sum_{i} e_{i}^{2} x f_{N}\left(x, Q^{2}\right)=\frac{Q^{2} \sigma\left(\gamma^{*} N\right)}{4 \pi^{2} \alpha_{\mathrm{em}}}$

With the transverse size of the $q \bar{q}$ pair frozen during the scattering, due to Lorentz contraction, the photon-nucleon cross section can be factorized:

$\sigma\left(\gamma^{*} N\right)=\int_{0}^{1} d z \int d^{2} r|\psi(z, r)|^{2} \sigma_{q \bar{q} N}(r)$

with the Sudakov variable $z$. The photon wave function $|\psi(z, r)|^{2}$ can be interpreted as the probability of finding a $q \bar{q}$ state with transverse separation $r$ and a momentum fraction $z$ and $(1-z)$ with respect to the virtual photon. The wave function can be expressed as [14]

$$
|\psi(z, r)|^{2}=\frac{3}{2} \frac{\alpha_{\mathrm{em}}}{\pi^{2}} \sum_{i=1}^{N_{f}} e_{i}^{2} P(z) a^{2} K_{1}(a r)^{2}
$$

where $P(z)=z^{2}+(1-z)^{2}$ is the splitting function of the gauge boson into the $q \bar{q}$ pair, $a^{2}=Q^{2} z(1-z)$ and $K_{1}$ is the modified Bessel function. The most important contributions come from the region where $a r$ is small $(a r \ll 1)$. Therefore pairs with a small transverse size are favored.

The cross section for the strong interaction of a small size configuration with the nucleon, which happens via gluon exchange, can be calculated in pQCD [15] to give

$$
\sigma_{q \bar{q} N}=\frac{\pi^{2}}{3} r^{2} \alpha_{s}\left(Q^{\prime 2}\right) x^{\prime} g_{N}\left(x^{\prime}, Q^{\prime 2}\right)
$$

One sees that the cross section for the interaction of the small pair is proportional to the size which is a consequence of color transparency. As a matter of fact $\sigma_{q \bar{q} N}$ decreases fast at short distances whereas the wave function and the gluon distribution strongly increase to compensate for this effect. The variables $x^{\prime}, Q^{\prime 2}$ are related to $z, x$, and $r^{2}$. The invariant mass squared of the pair is

$M^{2} \approx \frac{k_{T}^{2}}{z(1-z)}$

where $k_{T}^{2} \approx 1 / r^{2}$ being the transverse momentum squared of the quark and antiquark, respectively. The virtuality of the gluon is the given by $Q^{\prime 2}=4 / r^{2}$ and $\quad x^{\prime}=M^{2} /(2 m \nu)=k_{T}^{2} /(z(1-z) 2 m \nu)=$ $x / a^{2} r^{2}$. Therefore, the strong scaling violation of $x^{\prime} g_{N}\left(x^{\prime}, Q^{\prime 2}\right)$ at small $r$ (i.e. large $Q^{\prime 2}$ ) in turn can compensate for the smallness of the pair (for further details see [14] and references therein). Changing the integration variable from $z$ to $x^{\prime}$ as $d z=-x d x^{\prime}$ / $\left(x^{\prime 2} Q^{\prime 2} r^{2}\right)$ one derives the exact expression for the sea quark density as

$$
x f_{N}\left(x, Q^{2}\right)=\frac{3}{4 \pi^{3}} x \int_{x}^{1} \frac{d x^{\prime}}{x^{\prime 2}} \int_{4 / Q^{2}}^{\infty} \frac{d r^{2}}{r^{4}} \sigma_{q \bar{q} N}\left(x^{\prime}, r^{2}\right)
$$

The main problem at this point arises from the interplay between hard and soft physics. In some region $4 / Q^{2} \leq r^{2} \leq 4 / Q_{0}^{2}$ perturbation theory should be valid. For $r^{2}>4 / Q_{0}^{2}$, i.e. for small $Q^{2}$, the non-perturbative part dominates. To use the presently available parametrizations of the free nucleon parton densities we choose a lower cut-off of $Q_{0}^{2}=0.4 \mathrm{GeV}^{2}$. It is obvious that we thereby completely neglect the non-perturbative input below this scale. However, in Ref. [13] it was shown that for $Q^{2} \leq 10^{-1} \mathrm{GeV}^{2}$ a saturation in the shadowing ratio sets in. As a result, the uncertainty by choosing a cut-off at $Q_{0}^{2}=0.4 \mathrm{GeV}^{2}$ is on the few percent level. Also, when comparing to experimental results, we find good agreement with the data.

In the case of the gluon distribution of the free nucleon one considers the scattering of a virtual colorless probe (e.g. the virtual higgs boson) which proceeds via the production of a gluon pair which then strongly interacts with the nucleon. With the 
momentum cut-off the gluon density can be written as

$x g_{N}\left(x, Q^{2}\right)=\frac{4}{\pi^{3}} \int_{x}^{1} \frac{d x^{\prime}}{x^{\prime}} \int_{4 / Q^{2}}^{4 / Q_{0}^{2}} \frac{d r^{2}}{r^{4}} \sigma_{g g N}\left(x^{\prime}, r^{2}\right)$

The main difference between the scattering of the $q \bar{q}$ pair and the $g g$ pair stems from the much larger cross section $\sigma_{g g N}=9 / 4 \sigma_{q \bar{q} N}$ which in turn leads to a much stronger gluon shadowing.

In the case of deep inelastic scattering off nuclei the same relations hold but with the respective cross sections for the scattering of the hadronic and gluonic fluctuations off the nucleus. For the sea quark distribution in the nucleus $A$ one has

$x f_{A}\left(x, Q^{2}\right)=\frac{3}{4 \pi^{3}} x \int_{x}^{1} \frac{d x^{\prime}}{x^{\prime 2}} \int_{4 / Q^{2}}^{4 / Q_{0}^{2}} \frac{d r^{2}}{r^{4}} \sigma_{q \bar{q} A}\left(x^{\prime}, r^{2}\right)$

and for the gluon the resulting equation is given by

$x g_{A}\left(x, Q^{2}\right)=\frac{4}{\pi^{3}} \int_{x}^{1} \frac{d x^{\prime}}{x^{\prime}} \int_{4 / Q^{2}}^{4 / Q_{0}^{2}} \frac{d r^{2}}{r^{4}} \sigma_{g g A}\left(x^{\prime}, r^{2}\right)$

As stated above, it is the long distance phenomenon of the hadronic fluctuation which causes the shadowing effect. For the small $x$, i.e. large $\lambda$, the fluctuation interacts with more than one nucleon inside the nucleus. As a result of this coherent interaction one finds that $\sigma\left(\gamma^{*} A\right)<A \sigma\left(\gamma^{*} N\right)$. The specific feature that is responsible for the shadowing effect is the fact that $\sigma_{h A} \neq A \sigma_{h N}$. Within Glauber theory in the eikonal limit one finds [16]

$\sigma_{h A}=2 \int d^{2} b\left(1-e^{-\sigma_{h N} T_{A}(b) / 2}\right)$

with the nuclear thickness function

$T_{A}(b)=\int d z \rho_{A}(b, z)=\frac{A}{\pi R_{A}^{2}} e^{-b^{2} / R_{A}^{2}}$

The integration can be done exactly for a Gaussian parametrization of $T_{A}(b)$. Here we will include only the double scattering contribution which was shown [14] to strongly dominate the overall shadowing ratio
$R_{G}=g_{N}\left(x, Q^{2}\right) / g_{A}$; the triple and higher scattering terms give a contribution that is only on the percent level and is therefore neglected here. We use $[13,17]$

$$
\begin{aligned}
\sigma_{h A}= & A \sigma_{h N}\left[1-A^{1 / 3} \frac{\sigma_{h N}}{8 \pi a^{2}} \frac{A-1}{A} \exp \left(-\frac{a^{2} A^{2 / 3}}{2 \lambda^{2}}\right)\right. \\
& +\ldots]
\end{aligned}
$$

with $a=1.1 \mathrm{fm}$.

\section{Results}

Based on the approximations above, namely double scattering contribution only, a lower momentum cut-off at $Q_{0}^{2}=0.4 \mathrm{GeV}^{2}$, due to the lack of information on the non-perturbative input, and by employing the Glück, Reya, Vogt parametrization [18] we derived the shadowing ratios for quarks and gluons at the typical semi-hard scale $Q=2 \mathrm{GeV}$ for ${ }^{207} \mathrm{~Pb}$ and ${ }^{40} \mathrm{Ca}$ (see Figs. 1 and 2 ). One clearly sees that gluons are much stronger shadowed than sea quarks at small $x$ which is due to the effects outlined above. This is a very important feature since at the soon available collider energies one will particularly test the small $x$ region $\left(x=p_{T} / \sqrt{s}\right.$ at midrapidity) which becomes increasingly important due to the strong gluonic component inside the free nucleon and therefore in the heavy nuclei. This feature has a

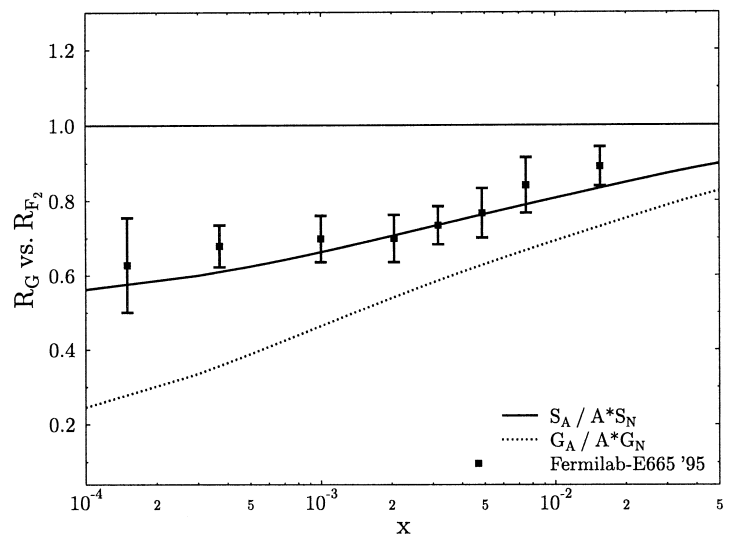

Fig. 1. $R_{F_{2}}$ versus $R_{G}$ at $Q^{2}=4 \mathrm{GeV}^{2}$ for ${ }^{207} \mathrm{~Pb}$. 


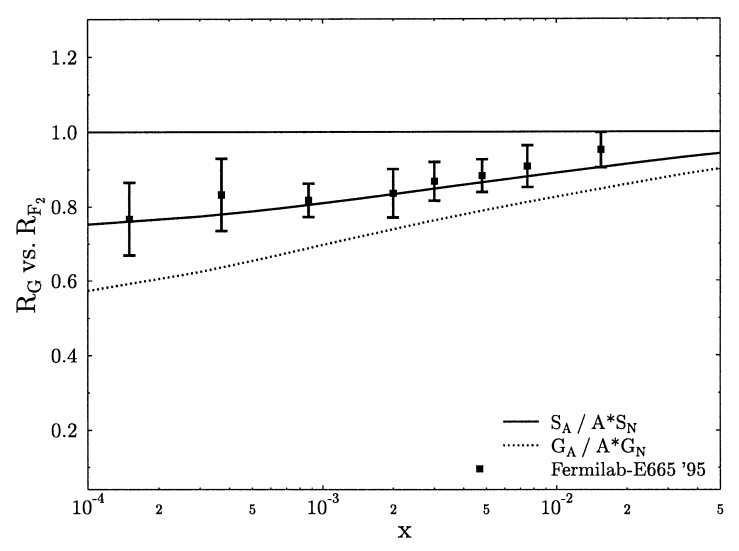

Fig. 2. $R_{F_{2}}$ versus $R_{G}$ at $Q^{2}=4 \mathrm{GeV}^{2}$ for ${ }^{40} \mathrm{Ca}$.

large impact on transverse energy production and on the initial temperature and entropy produced in heavy ion collisions. It should therefore come as no surprise when the final particle multiplicities are much lower than expected from calculations using e.g. the quark shadowing ratio $R_{F_{2}}(x)$ shown in Fig. 3 for gluons also. To check our results, we compared the results for $R_{F_{2}}(x)$ to Fermilab-E665 data [19] and find a good agreement. Since $s \simeq Q^{2} / x$, for deep inelastic events, one is restricted to a certain $Q^{2}$-range for the different $x$-values in the experiment. To account for this feature we compared to the data within the range $\left\langle Q^{2}\right\rangle \approx 2.42 \mathrm{GeV}^{2}-4.45 \mathrm{GeV}^{2}$ because our calculation was done at fixed $Q^{2}=4$ $\mathrm{GeV}^{2}$. One also sees that the agreement with the data is only good at small enough $x$, i.e. at large enough coherence lengths $\lambda \sim 1 / \Delta k_{z}$, where $\Delta k_{z}=$ $k_{z}^{\gamma}-k_{z}^{h}$ is the phase shift between the photon and the hadronic fluctuation. To derive the shadowing ratio for the complete structure function $F_{2}$ one could in principle improve the large $x$ behavior by inserting a splitting function $P_{g q}=z^{2}+(1-z)^{2}$ as suggested in Ref. [14] where $z=x / x^{\prime}$. Also one has to include the valence quark distribution as $F_{2}^{N}\left(x, Q^{2}\right)=$ $\sum_{i} e_{i}^{2}\left[x f_{N}\left(x, Q^{2}\right)+x v_{i}\left(x, Q^{2}\right)\right]$. But we here neglect this point since we were mainly interested in the region of small $x$. Some few comments concerning the connection of shadowing to diffractive photoproduction of hadrons from free nucleons are appropriate here. As is known, one can relate the coherent interaction part $\delta \sigma_{\gamma^{*} A}$ in $\sigma_{\gamma^{*} A}=A \sigma_{\gamma^{*} N}+\delta \sigma_{\gamma^{*} A}$ (leading to shadowing) to the diffractive production of states of mass $M_{x}$ from single nucleons [20-27]:

$\delta \sigma_{\gamma^{*} A} \sim-\int d M_{X}^{2} \frac{d \sigma_{\gamma^{*} N}^{\text {diff }}}{d M_{x}^{2} d t}(t \approx 0)$

The shadowing ratio can then be directly connected to the diffraction part of the free nucleon cross section:

$R_{A} \approx 1-C A\left(\frac{B}{\left\langle r^{2}\right\rangle_{A}}\right) \frac{\sigma_{\gamma^{*} N}^{\text {diff }}}{\sigma_{\gamma^{*} N}}$

where $C=3$ for Gaussian nuclear densities and $B=$ $7 \mathrm{GeV}^{-2}$ [21]. With the recent HERA data on diffractive production [28-30] one can therefore derive the shadowing ratio from measurements of free nucleon cross sections. This gives us in turn the possibility to check whether our results for the shadowing ratios coincide with the results derived by the approach using the HERA input, i.e. to see whether our results are consistent with the HERA diffraction data. If we compare our calculations for $R_{F_{2}}$ for ${ }^{40} \mathrm{Ca}$ with the results of $[20,21]$ we find very good agreement when corrected for the different $Q^{2}$ values. However, the absolute magnitude of shadowing of gluons appears to be smaller in the diffraction approach in Ref. [20] whereas our results indicate a much stronger gluon shadowing. One should however mention here that the results for $R_{G}$ using diffraction are sensitive to the gluonic distribution in the Pomeron which is only poorly known at present.

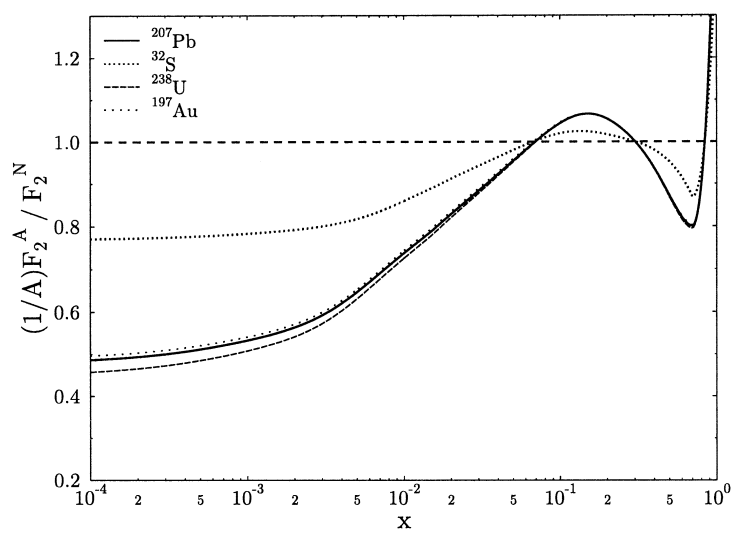

Fig. 3. $R_{F_{2}}=F_{2}^{A} / A F_{2}^{N}$ as parametrized by Eskola in Ref. [5] at $Q^{2}=4 \mathrm{GeV}^{2}$. 


\section{Conclusions}

We calculated the shadowing of sea quarks and gluons at small values of $x$ in the generalized vector meson dominance model (GVMD) approach. Based on the Glauber-Gribov multiple scattering picture for a $q \bar{q}$ pair with a coherence length $1 / \Delta k_{z}=\lambda \approx$ $1 / 2 m x$, we showed that one should expect significant differences between $R_{F_{2}}$ and $R_{G}$ at small $x$. The comparison to results derived by employing the diffractive production of hadrons from free nucleons and the latest HERA results show a very good agreement. The strong gluon shadowing, often neglected in the past, has severe consequences on quantities such as charmonium production, minijets and therefore on the production of transverse energy and entropy. When applying the much stronger gluon shadowing one should expect significantly smaller multiplicities for processes involving gluons in the initial state compared to calculations that accounted for all the shadowing effects by simply employing the quark shadowing ratio $R_{F_{2}}=F_{2}^{A} / A F_{2}^{N}$ for all parton species.

\section{References}

[1] EM Collaboration, J. Ashman, Phys. Lett. B 202 (1988) 603; M. Arneodo et al., Phys. Lett. B 211 (1988) 493.

[2] M. Arneodo et al., Nucl. Phys. B 333 (1990) 1.
[3] NM Collaboration, P. Amaudruz et al., Z. Phys. C 51 (1991) 387.

[4] N. Hammon, L. Gerland, H. Stöcker, W. Greiner, hepph/9807546, submitted to Phys. Rev. C.

[5] K.J. Eskola, Nucl. Phys. B 400 (1993) 240.

[6] N. Hammon, A. Dumitru, H. Stöcker, W. Greiner, Phys. Rev. C 57 (1998) 3292.

[7] B. Badelek, J. Kwiecinski, Rev. Mod. Phys. 64 (1992) 927; M. Arneodo, Phys. Rep. 240 (1994) 301.

[8] J. Qiu, Nucl. Phys. B 291 (1986) 746; A. Mueller, J. Qiu, Nucl. Phys. B 268 (1986) 427; F. Close, J. Qiu, R. Roberts, Phys. Rev. D 40 (1989) 2820.

[9] J. Kwiecinski, Z. Phys. C 45 (1990) 461.

[10] S. Kumano, Phys. Rev. C 48 (1993) 2016.

[11] S. Kumano, Phys. Lett. B 298 (1993) 171.

[12] G. Altarelli, G. Parisi, Nucl. Phys. B 126 (1977) 298.

[13] G. Piller, W. Ratzka, W. Weise, Z. Phys. A 352 (1995) 427.

[14] Z. Huang, H.J. Lu, I. Sarcevic, hep-ph/9705250.

[15] B. Blattel, G. Baym, L. Frankfurt, M. Strikman, Phys. Rev. Lett. 70 (1993) 896; L. Frankfurt, G. Miller, M. Strikman, Phys. Lett. B 304 (1993) 1.

[16] T. Bauer, R. Spital, D. Yennie, F. Pipkin, Rev. Mod. Phys. 50 (1978) 261.

[17] V.N. Gribov, JETP 30 (1970) 709.

[18] M. Glück, E. Reya, A. Vogt, Z. Phys. C 67 (1995) 433.

[19] Fermilab-E665 Collaboration, Z. Phys. C 67 (1995) 403.

[20] A. Capella et al., Europ. Physics. Journal C 5 (1998) 111.

[21] G. Piller, L. Ferreira, W. Weise, hep-ph/9812341.

[22] G. Piller, G. Niesler, W. Weise, Z. Phys. A 358 (1997) 407

[23] J. Kwiecinski, B. Badelek, Phys. Lett. B 208 (1988) 508.

[24] L. Frankfurt, M. Strikman, Nucl. Phys. B 316 (1989) 340.

[25] N.N. Nikolaev, B.G. Zakharov, Z. Phys. C 49 (1991) 607.

[26] W. Melnichouk, A. Thomas, Phys. Lett. B 317 (1993) 437.

[27] V. Barone, M. Genovese, Phys. Lett. B 412 (1997) 143.

[28] ZEUS Collaboration, M. Derrick, Z. Phys. C 63 (1994) 391.

[29] H1 Collaboration, S. Aid et al., Z. Phys. C 69 (1995) 27.

[30] H1 Collaboration, C. Adloff et al., Z. Phys. C 74 (1997) 221. 